\title{
A Pilot Study on the Diagnostic Accuracy of Proadrenomedullin and Proatrial Natriuretic Peptide in Lower Respiratory Tract Infections
}

\author{
Agustín Ruiz-González ${ }^{*}, 1$, Aureli Esquerda ${ }^{2}$, José M. Porcel ${ }^{1}$, Silvia Bielsa ${ }^{1}$, \\ Horacio Valencia ${ }^{1}$, Gonzalo $\mathrm{Cao}^{2}$ and Miquel Falguera ${ }^{1}$ \\ ${ }^{I}$ Department of Internal Medicine, Arnau de Vilanova University Hospital, IRBLLEIDA, Lleida, Spain \\ ${ }^{2}$ Department of Laboratory Medicine, Arnau de Vilanova University Hospital, IRBLLEIDA, Lleida, Spain
}

\begin{abstract}
Background: Pneumonia is the leading cause of death among infectious diseases in developed countries. However, the severity of pneumonia requiring hospitalization often makes the initial diagnosis difficult because of an equivocal clinical picture or interpretation of the chest film. The objective of the present study was to assess the usefulness of the plasma levels of mid-regional proadrenomedullin (MR-proADM) and mid-regional proatrial natriuretic peptide (MR-proANP) in differentiating pneumonia from other lower respiratory tract infections (LRTIs).
\end{abstract}

Methods: A retrospective study was conducted. The plasma levels of MR-proADM and MR-proANP were measured in 85 patients hospitalized for LRTIs, 56 of whom with diagnosis of pneumonia and 29 with other LRTIs.

Results: The patients with pneumonia had increased MR-proADM levels (median $1.46 \mathrm{nmol} / \mathrm{L}$ [IQR 25-75, 0.82-2.02 $\mathrm{nmol} / \mathrm{L}]$ ) compared with the patients with other LRTIs (median $0.88 \mathrm{nmol} / \mathrm{mL}$ [0.71-1.39 nmol/L]) $(\mathrm{p}=0.04)$. However, the MR-proANP levels did not show differences between the groups. The optimal threshold of MR-proADM to predict pneumonia was $1.5 \mathrm{nmol} / \mathrm{L}$, which yielded a sensitivity of 51.7\% (95\% CI, 38.0-65.3), a 79.3\% specificity (95\% CI, 60.3$92.0)$, and an odds ratio of 6.64 (95\% CI, 1.32-32.85). The combination of this parameter with C-reactive protein in an "and" rule increased the specificity for detecting pneumonia significantly.

Conclusion: MR-proADM levels (but not MR-proANP levels) are increased in patients with pneumonia although its discriminatory power is moderate.

Keywords: Adrenomedullin, atrial natriuretic peptide, biomarkers, diagnosis, pneumonia.

\section{INTRODUCTION}

Pneumonia is the sixth leading cause of death in general and the first among all infectious diseases in developed countries [1-3]. However, discriminating pneumonia from other chest infections where antibiotics are not required (i.e. acute bronchitis), particularly in its early stages, is sometimes difficult. In older patients, the clinical presentation is unclear, and interpreting chest radiographs can be difficult in patients with severe disease or previous pulmonary disease $[4,5]$.

The search for biomarkers to serve as diagnostic tools for clinicians has not yet produced a reliable tool. One biomarker of interest is mid-regional proadrenomedullin (MR-proADM), a more stable mid-region fragment of the parent precursor adrenomedullin [6]. Adrenomedullin is a peptide that is produced by multiple tissues types; it has pluripotent functions, including vasodilatory, antimicrobial, and anti-inflammatory activities [7]. In animal models of

*Address correspondence to this author at the Department of Internal Medicine, Arnau de Vilanova University Hospital, IRBLLEIDA. C/ Rovira Roure 80. 25198 Lleida, Spain; Tel: +34 973 248100;

Fax: +34 973 288754.; E-mail: agustinruiz@saludalia.com sepsis, exogenous adrenomedullin reduced acute lung injury, vascular permeability, and death [8-10]. On the other hand, mid-regional proatrial natriuretic peptide (MR-proANP) is a more stable mid-region fragment precursor of atrial natriuretic peptide, and the stimuli for its production most likely include proinflammatory factors and hypoxia. Its expression has been found in many tissues, but it is mainly produced in the atrium of the heart [11-12]. In previous studies, MR-proADM and MR-proANP levels have correlated with severity of pneumonia and death [13-17]. However, data on the diagnostic usefulness of these biomarkers is lacking in the literature.

Therefore, we examined the MR-proADM and MRproANP levels in a cohort of patients with lower respiratory tract infections (LRTIs) requiring hospitalization. We sought to describe the pattern of the initial biomarkers in pneumonia and other LRTIs, and to assess the potential diagnostic role in pneumonia.

\section{MATERIALS AND METHODS}

\section{Study Sample}

Since 2010, consecutive patients over age 18 with LRTIs requiring hospital admission were asked to provide 
additional blood samples for investigation. The study protocol was approved by the local ethical committee, and all of the participants provided written informed consent for the collection and storage of blood upon admission. Plasma was obtained by centrifugation and stored frozen at $-80^{\circ} \mathrm{C}$. For the present study, 85 plasma samples were randomly selected.

\section{Diagnostic Criteria}

LRTI was defined by the presence of at least one respiratory symptom (e.g., cough, sputum production, dyspnea, tachypnea, pleuritic pain) plus at least one finding during auscultation (i.e., rales) or one sign of infection (temperature $>38.0^{\circ} \mathrm{C}$, shivering, leukocyte count $>10$ or $<4$ $\mathrm{x} 10^{9}$ cells) regardless of antibiotic use. For CAP, a new infiltrate on the chest radiograph was also required. COPD was defined by post-bronchodilator spirometric criteria according to the Global initiative for Chronic Obstructive Lung Disease (GOLD)-guidelines as a FEV1/FVC ratio < $70 \%$. Acute bronchitis was defined as a LRTI in the absence of an underlying lung disease or focal chest signs and infiltrates on chest X-rays [18, 19]. A panel of three specialists had been previously reviewed chest X-rays from the patient population. Panel consensus in classifying LRTI was required for study inclusion.

The exclusion criteria were: 1) severe immunosuppression (HIV infection or severe haematological diseases); 2) immunosuppressive therapy (prednisone or an equivalent dose of $20 \mathrm{mg}$ daily for 2 weeks or any immunosuppressive regimen (azathioprine, cyclosporine, cyclophosphamide and/or other immunosuppressant drugs); 3) leukopenia $\left(<1,000\right.$ leukocytes per $\left.\mathrm{mm}^{3}\right)$ or neutropenia $(<500$ neutrophils per $\mathrm{mm}^{3}$ ) and/or chemotherapy in the previous year; 4) pulmonary abscess (radiological cavitation), aspiration pneumonia and obstructive pneumonia; 5) possible nosocomial origin (30 days from hospital discharge); and 6) known active neoplasm.

\section{Data and Severity Assessment}

The patients' clinical parameters were included in an electronic database. Comorbidities were documented and defined as the presence of one or more of the following: congestive heart failure, chronic obstructive pulmonary disease, chronic renal disease, chronic liver disease, cerebrovascular disease, malignancy, or diabetes mellitus. The microbiological work-up in the patients with pneumonia included sputum and/or bronchoalveolar lavage fluid samples for the Gram stain and culture, a urine sample for detecting Legionella pneumophila and Streptococcus pneumoniae antigens, and serological testing for Mycoplasma pneumoniae and Chlamydophila pneumoniae. The detection of respiratory pathogens was performed according to standard methods. In patients with severe exacerbations of COPD, a sputum sample was collected for the Gram stain and sputum culture. No microbiological tests were performed in the patients with acute bronchitis.

The severity assessment was made with the CURB-65 score and performed upon admission to the emergency department. The CURB-65 score consists of five variables: confusion, urea, respiratory rate $\geq 30$ breaths $/ \mathrm{min}$, blood pressure (systolic) $<90 \mathrm{~mm} \mathrm{Hg}$ or diastolic $\leq 60 \mathrm{mmHg}$, and age $\geq 65$ years [20]. One point is given for each parameter present, which results in CURB-65 scores of 0-5. The criteria for ICU admission were based on the American Thoracic Society (ATS) guidelines [21]. In brief, ICU admission was considered in patients with severe CAP, which was defined as the presence of one of two major criteria (the need for mechanical ventilation or septic shock), the presence of two of three minor criteria (systolic blood pressure $<90 \mathrm{mmHg}$, multilobar disease, or $\mathrm{PaO} 2 /$ FIO2ratio $<250$ ) or more than two CURB points. For COPD patients, the ICU criteria included severe acidosis or respiratory failure ( $\mathrm{pH}<7.25, \mathrm{pO} 2<40 \mathrm{mmHg}$ ), no response to initial treatment in the emergency department or worsening mental status (confusion, coma) despite adequate therapy [19].

\section{Laboratory Measurements}

Both MR-proADM and MR-proANP were measured in duplicated in stored EDTA plasma samples by the TRACE ${ }^{\circledR}$ technology, as previously described $[22,23]$. The procedure was fully automated in a KRYPTOR Compact analyzer (B.R.A.H.M.S. AG, Hennigsdorf, Germany). The other biochemical determinations were routinely performed as part of the admission study using conventional laboratory methods.

\section{Statistical Analysis}

The categorical variables were expressed as counts (percentages), and the continuous variables were expressed as medians and interquartile ranges (IQR), as appropriate. The $\chi 2$ and Fisher's exact tests were used, as appropriate, to compare the qualitative variables. In the case of quantitative variables, the Mann-Whitney U-test was used.

To assess the accuracy of the biomarkers for predicting the presence of pneumonia, we generated receiver operating characteristic curves and determined the area under the curve (AUC) and its $95 \%$ confidence interval $(95 \% \mathrm{CI})$. For each ROC curve, a cut-off point was determined as the value of the biomarker that maximised the sum of the sensitivity and specificity for diagnosing pneumonia. Pairwise comparisons of the AUC were conducted, following the Hanley and McNeil procedure. To adjust for confounders, a logistic regression model estimated the simultaneous impact of CRP and MR-proADM, along with significant variables in the univariate analysis predicting pneumonia. Associations were considered to be statistically significant if the p-value was $<0.05$. The statistical software packages used were SPSS 15.0 (SPSS Inc., Chicago, IL, USA) and MedCalc version 12.3.0 (MedCalc, Mariakerke, Belgium).

\section{RESULTS}

\section{Baseline Characteristics}

A total of 85 patients who were hospitalized for LRTIs were recruited; 56 were diagnosed with pneumonia and 29 with other LRTIs (13 with acute exacerbation of chronic obstructive pulmonary disease and 16 with acute bronchitis). The mean patient age was 71.0 years (range 20-96); 69.4\% were males, and $62.4 \%$ had comorbidities. Of the participants, $8.2 \%$ arrived from nursing home facilities. The patients with pneumonia had a median CURB65 score of 2.5. During the follow-up, 3 (3.5\%) patients were transferred to the ICU, and $7(8.2 \%)$ died (6 with pneumonia and 1 with other LRTIs). The mean in-patient stay was 8.4 days (range 2-41). 
Microbiological work-up were performed in patients with pneumonia or with severe exacerbations of COPD. A final etiological diagnosis was established in $29(42.0 \%)$ patients. The microorganisms identified were: Streptococcus pneumoniae (19), Chlamydophila pneumoniae (6), Legionella pneumophila, Staphylococcus aureus, Escherichia coli, and Aspergillus fumigatus (1 each).

\section{Data Analysis}

A comparison of the clinical, analytical and follow-up data between the groups is shown in Table 1. In a univariate analysis, 5 variables were different between the patients with pneumonia and those with other LRTIs: acute onset of symptoms (48\% vs 14\%), heart rate (100 vs 89 beats/min), the presence of rales (64\% vs 14\%), CRP (222 vs $102 \mathrm{mg} / \mathrm{L})$ and MR-proADM (1.46 vs $0.88 \mathrm{nmol} / \mathrm{L})$. However, in the multivariate analysis, the presence of rales, CRP and MRproADM maintained significant differences only.

Fig. (1) shows MR-proANP levels in the study population. The patients with pneumonia had similar MRproANP levels (median 233 pmol/L [IQR 25-75, 115-398 pmol/L]) compared with those with other LRTIs (median
$259 \mathrm{pmol} / \mathrm{L}$ [110-399 $\mathrm{pmol} / \mathrm{L}])(\mathrm{p}=0.88)$. Conversely, the patients with pneumonia had increased MR-proADM levels (median $1.46 \mathrm{nmol} / \mathrm{L}$ [IQR 25-75, $0.82-2.02 \mathrm{nmol} / \mathrm{mL}]$ ) compared with the patients with other LRTIs (median 0.88 $\mathrm{nmol} / \mathrm{L}$ [0.71-1.39 nmol/L]) (p=0.04) (Fig. 2).

The AUC for MR-proADM to discriminate between the patients with pneumonia and other LRTIs was 0.65 (95\% CI, 0.53-0.76), which was not inferior to the result obtained for CRP $(0.78$ [95\% CI, 0.67-0,86], $\mathrm{p}=0.11)$. The optimal threshold (minimal false negative and false positive results) of MRproADM to predict pneumonia was $1.5 \mathrm{nmol} / \mathrm{L}$, which yielded a sensitivity of $51.7 \%$ (95\% CI, 38.0-65.3), a $79.3 \%$ specificity (95\% CI, 60.3-92.0). As Table 2 shows, combining MRproADM with CRP in an "and" rule increased the specificity to $96 \%(95 \% \mathrm{CI}, 82-99)$ and the likelihood ratio positive to 9.2 (95\% CI, 1.3-65.1) for detecting pneumonia.

\section{DISCUSSION}

In the present study, MR-proADM levels but not MRpro-ANP levels were found to be increased in patients with pneumonia compared with other LRTIs.

Table 1. Comparison between the patients with pneumonia and those with other lower respiratory tract infections (LRTIs).

\begin{tabular}{|c|c|c|c|c|}
\hline \multicolumn{5}{|l|}{ Clinical findings } \\
\hline Days of illness & $3(2-5)$ & $5(3-7)$ & 0.14 & \\
\hline Previous antibiotic treatment & $14(25)$ & $11(38)$ & 0.21 & \\
\hline Cough & $46(82)$ & $24(82)$ & 0.94 & \\
\hline Sputum & $26(46)$ & $14(48)$ & 0.87 & \\
\hline Pleuritic chest pain & $20(36)$ & $6(21)$ & 0.15 & \\
\hline \multicolumn{5}{|l|}{ Physical findings } \\
\hline Systolic blood pressure (mmHg) & $118(91-139)$ & $127(108-142)$ & 0.20 & \\
\hline Heart rate (beats/min) & $100(80-111)$ & $89(77-101)$ & 0.03 & 0.08 \\
\hline Respiratory rate (breaths/min) & $28(20-36)$ & $24(24-29)$ & 0.97 & \\
\hline Rales & $36(64)$ & $4(14)$ & 0.00 & 0.01 \\
\hline \multicolumn{5}{|l|}{ Laboratory } \\
\hline WCC (white cell count) $\left(\times 10^{9}\right)$ & $12.3(7.7-17.3)$ & $11.5(7.7-14.4)$ & 0.37 & \\
\hline Haematocrit \% & $41(36-45)$ & $41(35-47)$ & 0.89 & \\
\hline Urea $\mathrm{mg} / \mathrm{dL}$ & $56(40-81)$ & $37(28-76)$ & 0.05 & \\
\hline
\end{tabular}

The data are expressed as the median (IQR) or N (\%). * Multivariate analysis. 


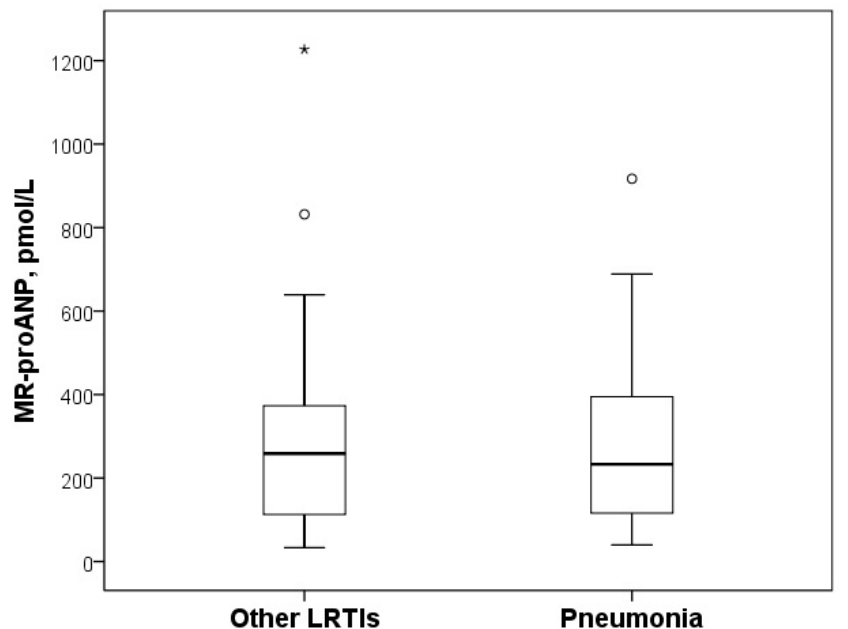

Fig. (1). Box plots of the MR-proANP plasma levels in the study population. The patients with pneumonia had similar MR-proANP levels (median 233 pmol/L [IQR 25-75, $115-398$ pmol/L]) compared with those with other LRTIs (median $259 \mathrm{pmol} / \mathrm{L}$ [110$399 \mathrm{pmol} / \mathrm{L}])(\mathrm{p}=0.88)$. The Box plots represent the $25^{\text {th }}$ and $75^{\text {th }}$ percentiles, with the internal horizontal lines showing the median. T-shaped bars represent the $10^{\text {th }}$ and $90^{\text {th }}$ percentiles. IQR, interquartile range; MR-proANP, mid-regional proatrial natriuretic peptide.

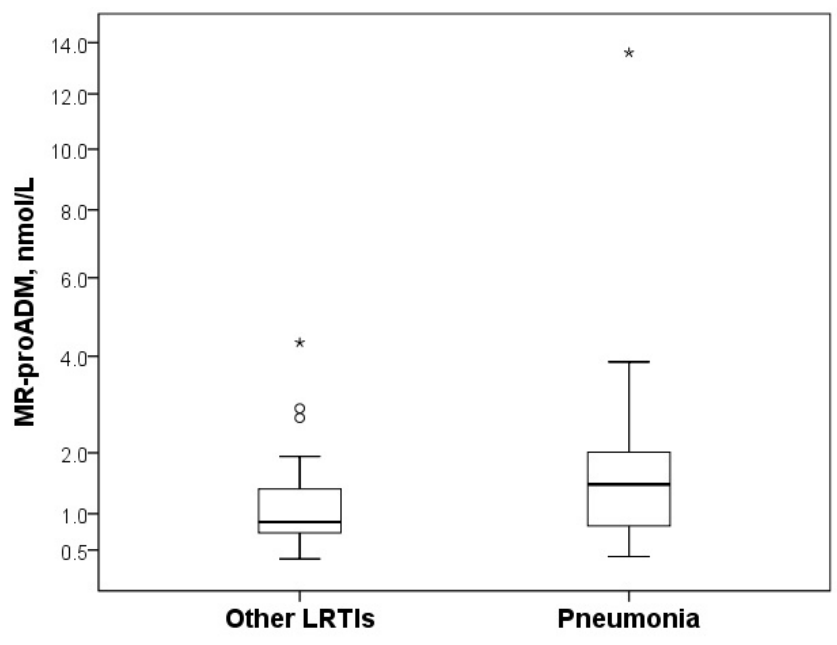

Fig. (2). Box plots of the MR-proADM plasma levels in the study population. The patients with pneumonia had increased MRproADM levels (median $1.46 \mathrm{nmol} / \mathrm{L}$ [IQR 25-75, 0.82-2.02 $\mathrm{nmol} / \mathrm{mL}$ ) compared with those with other LRTIs (median 0.88 $\mathrm{nmol} / \mathrm{L}$ [0.71-1.39 nmol/L]) ( $\mathrm{p}=0.04)$. The MR-proADM units are expressed as natural logarithm. The box plots represent the $25^{\text {th }}$ and $75^{\text {th }}$ percentiles, with the internal horizontal lines showing the median. T-shaped bars represent the $10^{\text {th }}$ and $90^{\text {th }}$ percentiles. IQR, interquartile range; MR-proADM, mid-regional proadrenomedullin.

The diagnosis of pneumonia is sometimes difficult in clinical practice. In older patients, the clinical presentation is unclear, and the accuracy of chest radiography, which remains the reference technique for diagnosing pneumonia, is $65 \%$ compared with a CT scan [24]. In addition, the search of biomarkers that could help in managing infections has produced conflicting results [25].
Table 2. MR-proADM characteristics alone or combined with C-reactive protein to predict pneumonia.

\begin{tabular}{|c|c|c|c|c|}
\hline Serum Markers & Sensitivity & Specificity & LR+ & LR- \\
\hline \hline $\begin{array}{c}\text { MR-proADM } \\
>1.5 \mathrm{nmol} / \mathrm{L}\end{array}$ & $\begin{array}{c}51.7 \\
(38.0-65.3)\end{array}$ & $\begin{array}{c}79.3 \\
(60.3-92.0)\end{array}$ & $\begin{array}{c}2.5 \\
(1.2-5.3)\end{array}$ & $\begin{array}{c}0.6 \\
(0.4-0.8)\end{array}$ \\
\hline $\mathrm{CRP}$ & 75.4 & 77.2 & 3.3 & 0.3 \\
$>150 \mathrm{mg} / \mathrm{L}$ & $(61.7-86.2)$ & $(54.6-92.2)$ & $(1.5-7.3)$ & $(0.2-0.5)$ \\
\hline $\mathrm{MR}-\mathrm{proADM}$ & 34.0 & 96.3 & 9.2 & 0.6 \\
$>1.5 \mathrm{nmol} / \mathrm{L}$ and & $(22.7-47.4)$ & $(81.7-99.3)$ & $(1.3-65.1)$ & $(0.5-0.8)$ \\
$\mathrm{CRP}>150 \mathrm{mg} / \mathrm{L}$ & & & & \\
\hline
\end{tabular}

The data are presented as \% $(95 \% \mathrm{CI})$. LR+: likelihood ratio positive; LR-: likelihood ratio negative.

In a study of 151 patients with systemic inflammatory response syndrome, the AUC for detecting a bacterial cause was 0.72 for procalcitonin (PCT), 0.74 for neutrophil count, and 0.81 for CRP [26]. In a study of 545 patients with LRTIs (373 with CAP), the AUCs of a clinical model, PCT and CRP were $0.79,0.76$ and 0.88 , respectively, to detect patients with pneumonia [27].

Both, MR-proANP and MR-proADM have been recently incorporated into the race of biomarkers primarily by adding prognostic information. Thus, in a study of 300 patients with pneumonia, the MR-proANP levels were significantly higher in the patients with high PSI risk class (IV-V) than in those with low PSI risk class (I-III) [13]. In a multicentre prospective study of 1,653 patients with pneumonia, MRproADM had a higher AUC for 30-day mortality than PCT (0.76 vs 0.65, respectively) [14]. In the multicentre ProHOSP study [15], the authors compared the prognostic accuracy of several biomarkers with the PSI and CURB65 clinical scores in 1,359 patients with LRTIs. The discriminatory power of biomarkers for predicting death ranged from an AUC of 0.60 for PCT to 0.76 for MR-proADM and 0.79 for MR-proANP. The CURB65 and PSI score had an AUC of 0.74 and 0.84 , respectively. In the multicentre CAPNETZ study [16], the authors compared the prognostic accuracy of several biomarkers with the PSI and CRB65 clinical scores in 728 patients with CAP. The AUC of MR-proADM for 28-day mortality $(0.85)$ was superior to those of MR-proANP $(0.81)$ and CRB-65 (0.72) for predicting mortality. Recently, MRproADM has shown a better discriminatory power than PSI score in identifying risk of death among CAP patients with PSI class IV and V (0.81 vs 0.66 , respectively) [28]. In addition, the utility of MR-proANP or MR-proADM have also been analysed to detecting the aetiology, the presence of bacteraemia, the risk of developing complications, or the need for ICU admission in patients with LRTIs [17, 29-31].

To the best of our knowledge, this is the first study analysing the diagnostic performance of MR-proANP and MR-proADM in an LRTI population. Our results supported the notion that high MR-proADM levels are found in patients with pneumonia compared with other LRTIs. At cutoff levels of $1.5 \mathrm{nmol} / \mathrm{L}$, the discriminatory power of MRproADM was moderate (sensitivity and specificity of approximately $52 \%$ and $80 \%$, respectively). However, the combination of MR-proADM with CRP (at cut-off levels of 
$150 \mathrm{mg} / \mathrm{L}$ ) in an "or/and" rule, significantly improved the operating characteristics. Indeed, the presence of any of both biomarkers showed a sensitivity of $86 \%$, whereas the presence of both biomarkers increased the specificity to $96 \%$ and the likelihood ratio positive to 9.2. By this rule, approximately $15 \%$ of patients with pneumonia were missed. However, the levels of MR-proANP did not differ between the groups. The fact that MR-proADM seems to be superior to MR-proANP might be explained by the multiple biological functions of adrenomedullin. Indeed, adrenomedullin is one of the most potent vasodilation agents with immune modulating, metabolic and bactericidal properties. In contrast, natriuretic peptides such as ANP correlate with the presence of heart of renal failure, which may be present only in the subgroup of patients with LRTIs and poor prognoses [6-12].

The present study has some limitations. First, the design was retrospective, and the effects of sample storage on the measurements have not been evaluated. Second, the population evaluated included adult patients with LRTIs who required hospital care; therefore, it may not be possible to extrapolate our conclusions to populations with mild LRTIs. Finally, immunosuppressed patients were excluded from the analysis, and this patient group has difficult diagnoses that could benefit from the use of biomarkers.

In our opinion, further studies with larger samples are needed to analyze the usefulness of MR-proADM testing alone or in combination with other biomarkers in the management of respiratory infections.

\section{CONCLUSION}

Among hospitalized patients with LRTIs, MR-proADM levels are increased in patients with pneumonia. The optimal threshold of MR-proADM to predict pneumonia was 1.5 $\mathrm{nmol} / \mathrm{L}$, which yielded a specificity of $79.3 \%$. MR-proANP levels did not show differences between groups.

\section{ABBREVIATIONS}

CAP $=$ Community-acquired pneumonia

LRTI $=$ Lower respiratory tract infection

MR-proADM $=$ Mid-regional proadrenomedullin

MR-proANP $=$ Mid-regional proatrial natriuretic peptide

\section{CONFLICT OF INTEREST}

The authors state that have no conflict of interest including any financial, personal or other relationships with other people or organizations with the submitted work that could inappropriately influence, or be perceived to influence, their work.

\section{ACKNOWLEDGEMENTS}

Declared none.

\section{REFERENCES}

[1] Anevlavis S, Bouros D. Community acquired bacterial pneumonia. Expert Opin Pharmacother 2010; 11: 361-74.

[2] Johansson N, Kalin M, Tiveljung-Lindell A, et al. Etiology of community-acquired pneumonia: increased microbiological yield with new diagnostic methods. Clin Infect Dis 2010; 50: 202-9.

[3] Restrepo MI, Anzueto A. Severe community-acquired pneumonia. Infect Dis Clin North Am 2009; 23: 503-20.
[4] Metlay JP, Schulz R, Yi-Hwei L, et al. Influence of age on symptoms at presentation in patients with community-acquired pneumonia. Arch Intern Med 1997; 157: 1453-59.

[5] Cortellaro F, Colombo $\mathrm{S}$, Coen $\mathrm{D}$, et al. Lung ultrasound is an accurate diagnostic tool for the diagnosis of pneumonia in the emergency department. Emerg Med J 2012; 29: 19-23.

[6] Struck J, Tao C, Morgenthaler NG. Identification of an adrenomedullin precursor fragment in plasma of sepsis patients. Peptides 2004; 25: 1369-72.

[7] Beltowski J, Jamroz A. Adrenomedullin: what do we know 10 years since its discovery? Pol J Pharmacol 2004; 56: 5-27.

[8] Itoh T, Obata $\mathrm{H}$, Mmurakami $\mathrm{S}$, et al. Adrenomedullin ameliorates lipopolysaccharide-induced acute lung injury in rats. Am J Physiol Lung Cell Mol Physiol 2007; 293: 446-52.

[9] Temmesfeld-Wollbrück B, Brell B, David I, Dorenberg M, et al. Adrenomedullin reduces vascular hyperpermeability and improves survival in rat septic shock. Intensive Care Med 2007; 33: 703-10.

[10] Shindo T, Kurihara H, Maemura K, et al. Hypotension and resistance to lipopolysaccharide-induced shock in transgenic mice overexpressing adrenomedullin in their vasculature. Circulation 2000; 101: 2309-16.

[11] Beishuizen A, Hartemink KJ, Vermes I, et al. Circulating cardiovascular markers and mediators in acute illness: an update. Clin Chim Acta 2005; 354: 21-34

[12] Levin ER, Gardner DG, Samson WK. Natriuretic peptides. N Engl J Med 1998; 339: 321-8.

[13] Prat C, Lacoma A, Dominguez J, et al. Midregional pro-atrial natriuretic peptide as a prognostic marker in pneumonia. J Infect 2007; 55: 400-7.

[14] Huang DT, Angus DC, Kellum J, et al. Midregional proadrenomedullin as a prognostic tool in community-acquired pneumonia. Chest 2009; 136: 823-31.

[15] Schuetz P, Wolbers M, Christ-Crain M, et al. Prohormones for prediction of adverse medical outcome in community-acquired pneumonia and lower respiratory tract infections. Critical Care 2010; 14: 2-14

[16] Krüger S, Ewig S, Giersdorf S, et al. Cardiovascular and inflammatory biomarkers to predict short- and long-term survival in community-acquired pneumonia. Results from the German competence network, CAPNETZ. Am J Respir Crit Care Med 2010; 182: 1426-34.

[17] Bello S, Lasierra AB, Minchole E, et al. Prognostic power of proadrenomedullin in community-acquired pneumonia is independent of aetiology. Eur Respir J 2012; 39: 1144-55.

[18] Woodhead M, Blasi F, Ewig S, et al. Guidelines for the management of adult lower respiratory tract infections. Eur Respir J 2005; 26: 1138-80

[19] From the global strategy for the diagnosis, management and prevention of cOPD, global initiative for chronic obstructive lung disease (GOLD) 2013. Available from: http://www.goldcopd.org/

[20] Lim WS, van der Eerden MM, Laing R, et al. Defining community acquired pneumonia severity on presentation to hospital: an international derivation and validation study. Thorax 2003; 58: 377-82.

[21] Mandell LA, Wunderink RG, Anzueto A, et al. Infectious diseases society of America/American thoracic society consensus guidelines on the management of community-acquired pneumonia in adults. Clin Infect Dis 2007; 44: S27-72.

[22] Morgenthaler NG, Struck J, Thomas B, et al. Immunoluminometric assay for the midregion of pro-atrial natriuretic peptide in human plasma. Clin Chem 2004; 50: 234-36.

[23] Morgenthaler NG, Struck J, Alonso C, et al. Measurement of midregional proadrenomedullin in plasma with an immunoluminometric assay. Clin Chem 2005; 51: 1823-9.

[24] Syrjala H, Broas M, Suramo I, et al. High-resolution computed tomography for the diagnosis of community-acquired pneumonia. Clin Infect Dis 1998; 27: 358-63.

[25] Blasi F, Bocchino M, Di Marco F, et al. The role of biomarkers in low respiratory tract infections. Eur J Intern Med 2012; 23: 429-35. Kofoed K, Andersen O, Kronborg G, et al. Use of plasma Creactive protein, procalcitonin, neutrophils, macrophage migration inhibitory factor, soluble urokinase-type plasminogen activator receptor, and soluble triggering receptor expressed on myeloid cells-1 in combination to diagnose infections: a prospective study. Crit Care 2007; 11: R38. 
[27] Müller B, Harbarth S, Stolz D, et al. Diagnostic and prognostic accuracy of clinical and laboratory parameters in communityacquired pneumonia. BMC Infect Dis 2007; 7:10.

[28] Courtais C, Kuster N, Dupuy AM, et al. Proadrenomedullin, a useful tool for risk stratification in high Pneumonia Severity Index score community-acquired pneumonia. Am J Emerg Med 2013; 31: 215-21.

[29] Guinard-Barbier S, Chenevier-Gobeaux C, Grabar S, et al. Bacteremia and MR-proANP changes in mild community-acquired pneumonia. Biomarkers 2011; 16: 637-41.
[30] Lacoma A, Rodríguez N, Prat C, et al. Usefulness of consecutive biomarkers measurement in the management of communityacquired pneumonia. Eur J Clin Microbiol Infect Dis 2012; 31: 825-33.

[31] Renaud B, Schuetz P, Claessens YE, et al. Proadrenomedullin improves REA-ICU score for predicting early severe communityacquired pneumonia. Chest 2012; 142: 1447-54.

(C) Ruiz-González et al.; Licensee Bentham Open.

This is an open access article licensed under the terms of the Creative Commons Attribution Non-Commercial License (http://creativecommons.org/licenses/by-nc/3.0/) which permits unrestricted, non-commercial use, distribution and reproduction in any medium, provided the work is properly cited. 